\title{
A novel mid-maturing cultivar with high dry matter content from seedlings of 'Jinfeng' kiwifruit (Actinidia chinensis)
}

\author{
Guanglian Liao ${ }^{1}$, Xiaobiao $\mathrm{Xu}^{1}$, Min Zhong ${ }^{1}$, Chunhui Huang ${ }^{1}$, Guiqing $\mathrm{Tu}^{2}$, Bangming $\mathrm{Li}^{2}$, Junjie $\mathrm{Tao}^{1}$, \\ Xueyan $\mathrm{Qu}^{1}$, Shanggao Zhao ${ }^{2}$ and Jianhua Leng ${ }^{2}$ \\ ${ }^{1}$ Kiwifruit Institute of Jiangxi Agricultural University, Nanchang Jiangxi, P.R. China \\ ${ }^{2}$ Kiwifruit Institute of Fengxin County in Jiangxi Province, Yichun Jiangxi, P.R. China
}

\section{Summary}

Dry matter is an extreme important index of fruit flavor quality, it affects flavor and texture of Actinidia. In 1979, 'Jinfeng' (code was '793'), a late-maturing cultivar with high yield and golden flesh was discovered. However, with the above advantages, its dry matter was low after we planted it at low altitudes (Fengxin county, $114^{\circ} 55^{\prime} \mathrm{E} ; 2^{\circ} 35^{\prime} \mathrm{N}, 70 \mathrm{~m}$ ) by vegetative propagation. In 2008, pollen from 'Ganxiong 1 ' (A. chinensis) was used to pollinate 'Jinfeng' through collaboration between Jiangxi Agricultural University and Kiwifruit Institute of Fengxin County in Jiangxi Province, collected seeds and sown in following year. A tetraploid genotype (code was 'GJ 3') with high dry matter had been found from 893 progenies. In addition, it not only retained the excellent characteristics of the female parent, but also matured one month earlier than 'Jinfeng'. We rafted its scions to asexual reproduction in Fengxin County (rootstock was A. chinensis) to carry out DUS test (Distinctness, Uniformity and Stability) in 2013-2015. The results showed that high dry matter was a stable characteristic; compared with the 'Jinfeng' and 'Hort16A', the dry matter content improved by $45.05 \%$ and $22.26 \%$. Meanwhile the fruit is wide ellipse and with golden flesh. The dry matter ranges from $19.2 \%$ to $21.2 \%$, soluble solids content (SSC) ranges from $16.8 \%$ to $19.1 \%$, and ascorbic acid (AsA) content is $86.3 \mathrm{mg} 100 \mathrm{~g}^{-1}$. The paper introduced the main agronomic traits of high dry matter cultivar and discussed the main reason of regulatory mechanism on sugar types during fruit growth, and its usefulness for studying the regulatory mechanism of dry matter in kiwifruit (A. chinensis).

\section{Keywords}

Actinidia chinensis, high dry matter, mid-maturing, sugar and acid compositions

\section{Introduction}

'Hort16A' kiwifruit, selected from New Zealand, was the main cultivar of $A$. chinensis grown around the world nowadays. Its success is based on its excellent taste, productivity and golden flesh. While the ability to resist canker of 'Hort$16 A^{\prime}$ kiwifruit is weak, bacterial canker was first observed on the kiwifruit 'Hort16A' in Jeju Province, Korea, in spring 2006. A sudden outbreak and rapid spread of the bacterial canker resulted in the death of severely infected vines and eradication of completely devastated orchards of the kiwifruit 'Hort16A' (Koh et al., 2010). At the same time, the main

\section{Significance of this study}

What is already known on this subject?

- Dry matter is an extreme important fruit quality parameter, affecting both flavor and texture of Actinidia.

What are the new findings?

- 'Fenghuang 1' kiwifruit is a novel tetraploid cultivar with a high dry matter content, high sugar and low acidity concentration, respectively. Moreover, the cultivar matures in mid-season and is more resistant to canker than 'Hort16A'.

What is the expected impact on horticulture?

- It is expected that the cultivar will lead to an improved understanding of the regulatory mechanism of dry matter in kiwifruit and extension of the supply period with fruit of $A$. chinensis.

use of such a single cultivar implies both possible genetic vulnerability problems, and more importantly, poor utilization of available genetic potential and limited choices for consumers and markert (Mavromatis et al., 2010). Research for new genetic material with high commercial value and strong resistance to canker is necessary. That occurs in all species, including kiwifruit (Atkinson and Macrae, 2007). Breeding programs are directed primarily toward large fruit size, good flavor, novel flesh color, hermaphroditism, variation in harvest period and improved yield (Seal, 2003). Dry matter is an important index of fruit flavor quality; it affects flavor and texture of Actinidia (Nardozza et al., 2011).

In 1979, A. chinensis 'Jinfeng' (code was '79-3', 4X), a late-maturing cultivar with high yield and golden flesh, was discovered at an altitude of $1,043 \mathrm{~m}$. The cultivar was grown commercially in Fengxin County from the 1980s. Although it had the characteristics of golden flesh, high yield and strong resistance and so on, it has been replaced by other cultivars due to the low SSC and dry matter content. Therefore, we hope to breed a novel germplasm with high fruit dry matter content and at the same time have the excellent characteristics of female parent 'Jinfeng' by crossbreeding. In 2008, pollen from 'Fengxiong 1 ' $(A$. chinensis, $4 \mathrm{X})$ was used to pollinate 'Jinfeng' through Jiangxi Agricultural University and Kiwifruit Institute of Fengxin County in Jiangxi Province, then collected seeds and sown in following year. A tetraploid genotype (code was 'GJ-3') with high dry matter, that had been found from 893 progenies. We found it not only retained the excellent characteristics of the female parent 'Jinfeng', but 


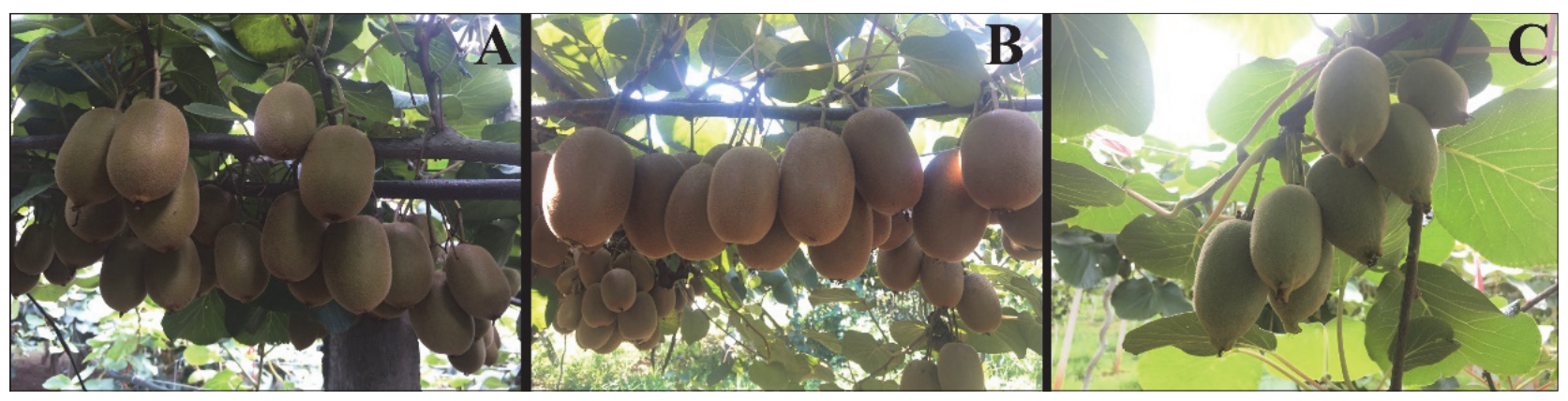

Figure 1. Fruit of 'Fenghuang 1' (A), 'Jinfeng' (B), and 'Hort16A' (C).

also matured about a week earlier than it. We sheared its scions to asexual reproduction in Fengxin County (rootstock was wild $A$. chinensis) to carry out DUS test (Distinctness, Uniformity and Stability) in 2015-2017. In 2014, the tetraploid genotype was named 'Fenghuang 1 '.

The discovery and analysis of a mid-maturing cultivar with high dry matter content would extend fruit supply period of $A$. chinensis, and contribute to our understanding of the regulatory mechanism of dry matter in A. chinensis and more generally in kiwifruit. The paper introduces the main agronomic traits of high dry matter cultivar and discusses the main reason of regulatory mechanism on sugar types during fruit growth, and its usefulness for studying the regulatory mechanism of dry matter in kiwifruit (A. chinensis).

\section{Materials and methods}

\section{Plant materials}

The research was conducted in a kiwifruit orchard located at Fengxin County, Jiangxi Province, where vines from the three compared kiwifruit materials were cultivated together. Three kiwifruit cultivars, 'Hort16A', 'Jinfeng' and 'Fenghuang 1 ', all belonging to the $A$. chinensis cultivar, were used for testing materials in this study; among them 'Hort16A' was identified as mid-maturing cultivar (matured in late-September), 'Jinfeng' was late-maturing cultivar (matured in late-October). In this study, we sheared the scions of test materials to asexual reproduction at Fengxin County (rootstock was wild A. chinensis) (Figure 1). Thirteen sampling stages determined by the days after full bloom (DAF) were as follows: 10 , $20,30,40,55,70,85,100,115,130,145,160$ and 175 DAF. Of the 50 fruits of each cultivar at each stage, 10 fruits were used to measure the physiological parameters. All fruit samples were taken from kiwifruit germplasm garden of Fengxin County Agricultural Bureau, Jiangxi Province, China. The flesh of the fresh samples was dissected using a blade, frozen immediately in liquid nitrogen, and then stored at $-80^{\circ} \mathrm{C}$.

\section{Morphological characteristics}

Difference in anatomy and morphology characteristics between three compared kiwifruit materials were determined. Twenty vines per genotype were examined and the main characteristics of leaves (shape, apex), flowers (color, size arrangement of petals) and fruits (size, shape) were been texted.

\section{Molecular genetic analysis}

Molecular genetic analysis for Actinidia genotypes was based on microsatellite DNA molecular markers using PCR (Huang et al., 1998). For the DNA genetic analysis, repeatable samples of the three compared kiwifruit materials were used. The process for DNA analysis included leaf sampling
$(0.1 \mathrm{~g})$ per genotype and DNA extraction process via a modified CTAB method. The extracted DNA was quantified on agarose gel by comparison with report samples (DNA markers). The DNA quality and quantity were tested for appropriate molecular genetic analysis. For genome screening, six primer pairs were used to amplify dinucleotide tandems AG/CT and AC/GT. The primers pairs were developed in multilateral INCO-DC project and are appropriate, accurate and reliable for Actinidia molecular genetic analysis. PCR products were separated in an 8\% polyacrylamide gel, $1.5 \mathrm{~mm}$ thick, and band visualization was possible by the silver nitrate method.

\section{Ploidy level analysis}

The young leaves of about $0.5 \mathrm{~cm}^{2}$ sample were put on the petri dishes, add $400 \mu \mathrm{L}$ extract liquor to it and use a sharp blade to cross cut the sample, soak the sample for 3 minutes, then filter the sample into the sample tube with the filter net, and add 1,600 $\mu$ l DAPI dye, wait for 3 minutes, we can use the CyFlow Cube flow cytometer to detect the ploidy. With the known multiplier 'Hongyang' (2X), 'Jinyan' (4X), 'Jinkui' $(6 \mathrm{X})$ as the standard sample, we set the voltage and flow rate before testing the ploidy of sample.

\section{Fruit quality evaluation during fruit development}

The weight of fruits was tested immediately after harvest. Fruit quality evaluation included hue angle of flesh color (color-difference meter model is Chromameter CR-400; the mean was taken from two measurements on opposite sides around the equator of each fruit immediately after careful skin removal), flesh firmness (two measurements per fruit with texture analyzer and $8 \mathrm{~mm}$ plunger), fruit dry matter (with slice thickness of $1 \mathrm{~mm}$ ), soluble solids content (with numerically explicit sugar meter). Finally, the content of soluble sugar, titratable acid, flavone, total phenols, pigment and ascorbic acid were measured by grinding the fruit pulp treated with liquid nitrogen (Gao, 2006; Wu et al., 2012). At the same time, the sugar and acid compositions were tested with High Performance Liquid Chromatography with reference to the method of peach (Niu et al., 2006).

Statistical analyses for physical and quality characteristics were performed with analysis of variance over the materials and period with the SPSS statistical package (SPSS 17.0). Significance levels are determined per parameter.

\section{Results}

Comparison of economic characters between the three materials

The comparative description of morphological characteristics between three materials for distinctness, is presented in Table 1. The most frequent leaf shape for 'Fenghuang 1' was determined as very broad ovate and the shape of fruits 
TABLE 1. Comparison of economic characters between the test materials. The same letters are not significantly different on test materials at $5 \%$ level.

\begin{tabular}{lccc}
\hline Characteristics & 'Fenghuang 1' & 'Jinfeng' & 'Hort16A' \\
\hline Leaf: shape & Very broad ovate & Ovoid & Broad ovate \\
Leaf: shape of apex & Acuminate & Acuminate & Acute \\
Flower: diameter & Medium & Medium & Medium \\
Flower: size & Medium & Medium & Medium \\
Flower: arrangement of petals & Overlapping & Overlapping & Overlapping \\
Fruits: average weight (g) & $93.09 \mathrm{c}$ & $110.31 \mathrm{a}$ & $100.21 \mathrm{~b}$ \\
Fruits: shape & Wide ellipse & Ellipse & Orbicular-ovate \\
Fruits: fruit shape index & $1.56 \mathrm{a}$ & $1.39 \mathrm{~b}$ & $1.57 \mathrm{a}$ \\
Fruits: soluble solids content (\%) & $17.9 \mathrm{a}$ & $11.01 \mathrm{c}$ & $15.56 \mathrm{~b}$ \\
Fruits: dry matter (\%) & $21.2 \mathrm{a}$ & $14.56 \mathrm{c}$ & $17.34 \mathrm{~b}$ \\
Fruits: hue of flesh color & $96.67 \mathrm{~b}$ & $97.08 \mathrm{~b}$ & $105.03 \mathrm{a}$ \\
Incidence of canker disease in the field & None & Little & Little \\
Time of beginning flowering (Month. Day) & $4.18-4.19$ & $4.27-4.28$ & $4.13-4.14$ \\
Time of harvest (Month. Day) & $9.27-9.28$ & $10.5-10.4$ & $9.23-9.24$ \\
\hline
\end{tabular}

TABLE 2. Polymorphic SSR molecular markers used to discriminate three materials.

\begin{tabular}{|c|c|c|c|c|c|}
\hline DNA primers & Sequence & $\begin{array}{l}\text { Molecular size } \\
\text { (bp) }\end{array}$ & 'Fenghuang 1' & 'Jingfeng' & 'Hort16A' \\
\hline A0 55 & $\begin{array}{l}\text { FOR 5'TCCATTCCGCCCATCCTT3' } \\
\text { FEV 5'GTCCGACATTCTTGTGGTTCTG3' }\end{array}$ & 150 & + & + & + \\
\hline UDK 96-019 & $\begin{array}{l}\text { FOR 5'ATACACTTGAAGCGCCGC3' } \\
\text { FEV 5'AAGCAGCCATGTCGATACG3' }\end{array}$ & 100 & + & - & + \\
\hline UDK 96-039 & $\begin{array}{l}\text { FOR 5'GGTTTGATCGGTCTTCGAAA3' } \\
\text { FEV 5'ATAAATGTGTGCCAGTGCGA3' }\end{array}$ & 50 & - & + & - \\
\hline UDK 96-035 & $\begin{array}{l}\text { FOR 5'AAGAGCCATAGCTTATTCACCG3' } \\
\text { FEV 5'AAGTAAAGCCATTGTCATTGCA3' }\end{array}$ & 100 & - & - & + \\
\hline UDK 96-040 & $\begin{array}{l}\text { FOR 5'TCGAGTTACCTAGCTACTCCGC3' } \\
\text { FEV 5'CAAGGGAAGAAAATGTTGAACC3' }\end{array}$ & 50 & + & + & - \\
\hline Est Ad-42 & $\begin{array}{l}\text { FOR 5'GTTAATTTGATCGGGATGG3' } \\
\text { FEV 5'GAGGAGCTTGAGCTGCTAT3' }\end{array}$ & 150 & + & - & - \\
\hline
\end{tabular}

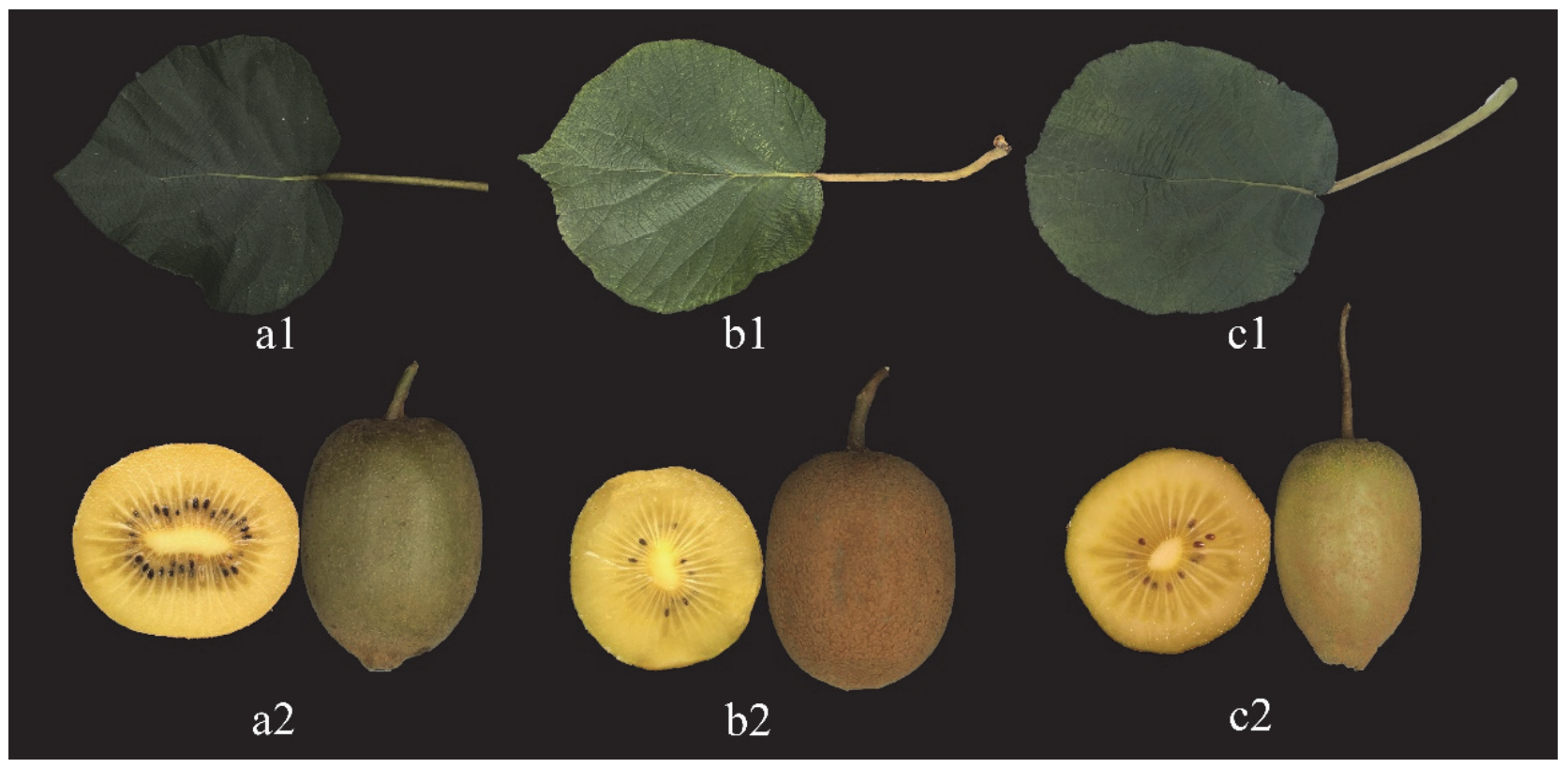

FIGURE 2. Morphology of leaf and fruits of three materials, a: ‘Fenghuang 1', b: 'Jinfeng', c: 'Hort16A'. 


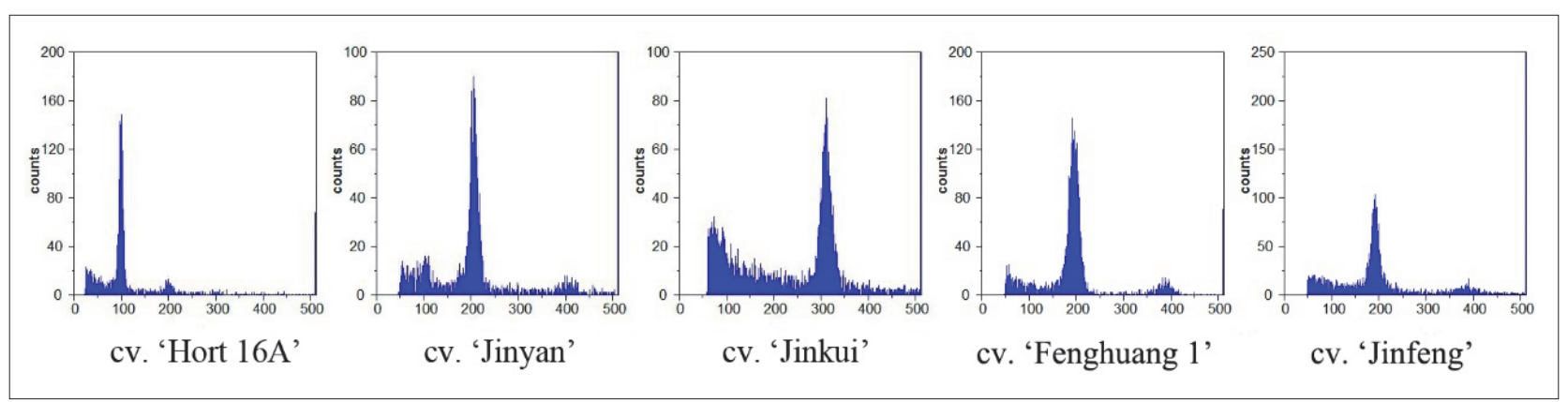

FIGURE 3. The data of ploidy test. With the known multiplier 'Hort16A' (2X), 'Jinyan' (4X), 'Jinkui' (6X) as the standard sample, set the voltage and flow rate before test the ploidy of sample.

was described as wide ellipse. There was no significant difference in flower characteristics (diameter, size and arrangement of petals) between the three materials, while the dry matter and soluble solids content of 'Fenghuang 1' were significantly higher than 'Jinfeng' and 'Hort16A', improved by $45.05 \%$ and $22.26 \%$. The time of beginning flowering and harvest was also different; the cultivar bloomed in the middle of April and was harvested in late September. The leaf color was described as dark green and the fruits characterized by smaller size and wide ellipse (Figure 2).

\section{Molecular and ploidy date}

The difference between the three materials according to their molecular profile as determined by presence and absence of alleles of the same molecular weight are shown in Table 2. The new kiwifruit cultivar was detected as tetraploid, which is inconsistent with 'Hort16A' (Figure 3).

\section{Dynamic changes of fruit quality during fruit growth and development}

1. Fruit weight. The single fruit weight of kiwifruit was measured during its fruit development. The growth period of fruit was $175 \mathrm{~d}$ and was formal "double S" curve; the first developing fruit period was DAFB $10 \mathrm{~d}-55 \mathrm{~d}$, the second was DAFB 70 d-175 d (Figure 4), the average single fruit weight was $88.87 \mathrm{~g}$ at the later stage of fruit development.

2. Soluble solid content and dry matter. The soluble solid content and dry matter of the fruit was measured only after DAF $100 \mathrm{~d}$, and the content was $5.40 \%$ and $8.35 \%$ at the time. At the DAF $115 \mathrm{~d}$, both decreased slightly and then increased. The soluble solid content and dry matter of the hard fruit was $13.00 \%$ and $17.53 \%$ (Figure 5); after ripening at room temperature, the content rose to $16.70 \%$ and $18.23 \%$.

3. Fruit firmness. The skin hardness and pulp hardness of the fruit were both slightly increased at DAF $100 \mathrm{~d}-115 \mathrm{~d}$, and both showed a downward trend after DAF $115 \mathrm{~d}$. The skin was much harder than the flesh, and the drop of flesh firmness was even more obvious (Figure 6).

4. Flesh color. The color of the transverse was different with the growth and development of the fruit, DAF $130 \mathrm{~d}$ was an important period, the transverse turned to yellow after this period. At the same time, the seed occurred at DAF $70 \mathrm{~d}$, and turned to brown at DAF $115 \mathrm{~d}$ (Figure 7). The color of flesh was determined, we found that the value of $h$ got smaller and smaller during DAF $55 \mathrm{~d}-130 \mathrm{~d}$, other values don't fluctuate much (Figure 8).

5. Titratable acid, soluble sugar and ascorbic acid content. The content of titratable acid of 'Fenghuang 1' showed a slow rising trend during the whole fruit development, the content rose from $0.895 \%$ to $1.769 \%$, the increase is small. While the content of titratable acid also showed a slow rising trend at DAF10 d-85 d, then showed a rapid upward trend at

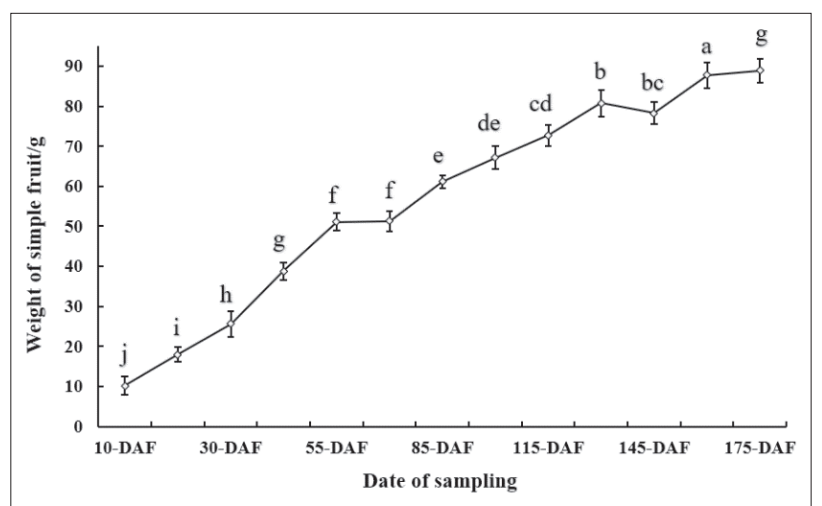

FIGURE 4. Changes of single fruit weight of 'Fenghuang 1' during its development.
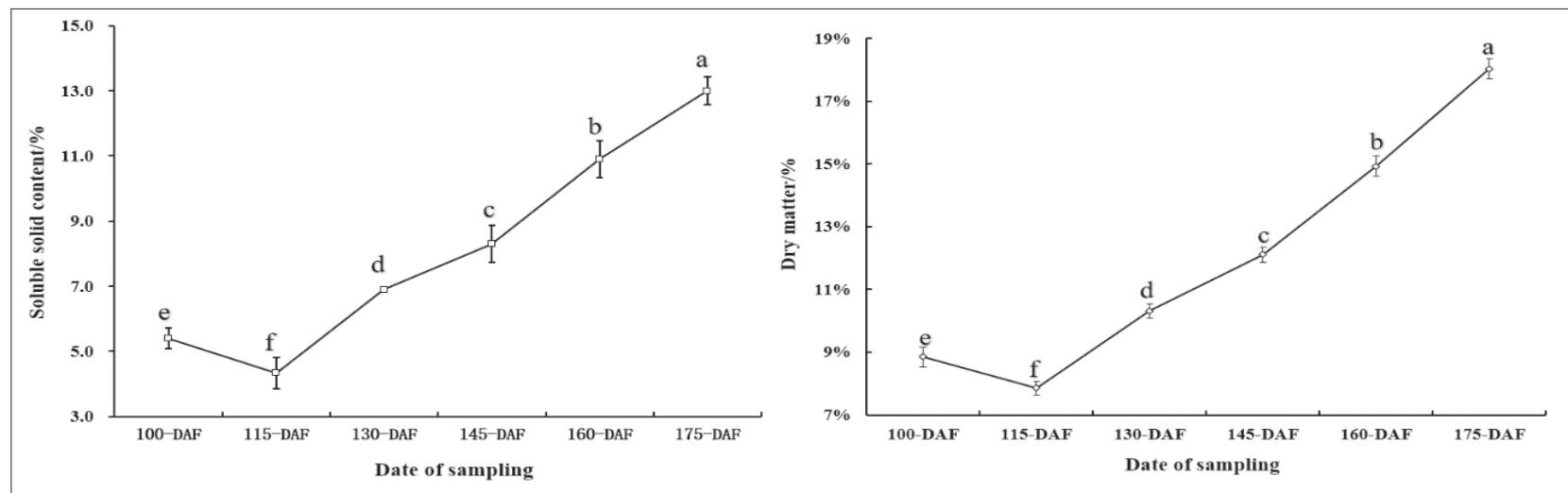

FigurE 5. Changes of soluble solid content and dry matter of 'Fenghuang 1' during its development. 


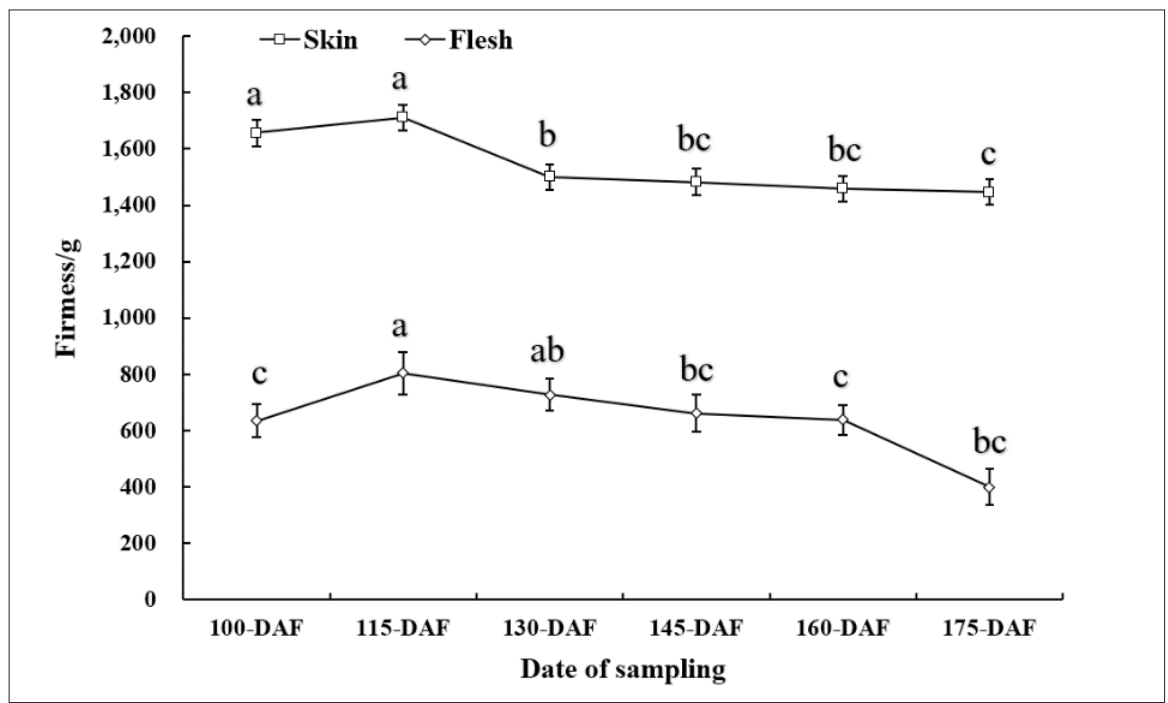

FIGURE 6. Changes of firmness and flesh of 'Fenghuang 1' during fruits development.

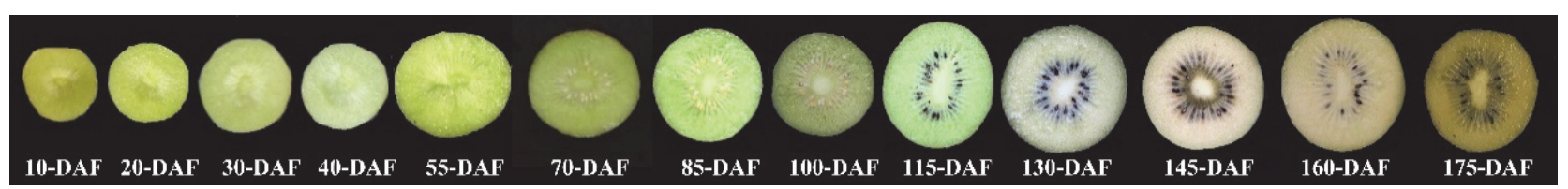

FigURE 7. Color of transverse during fruits development.

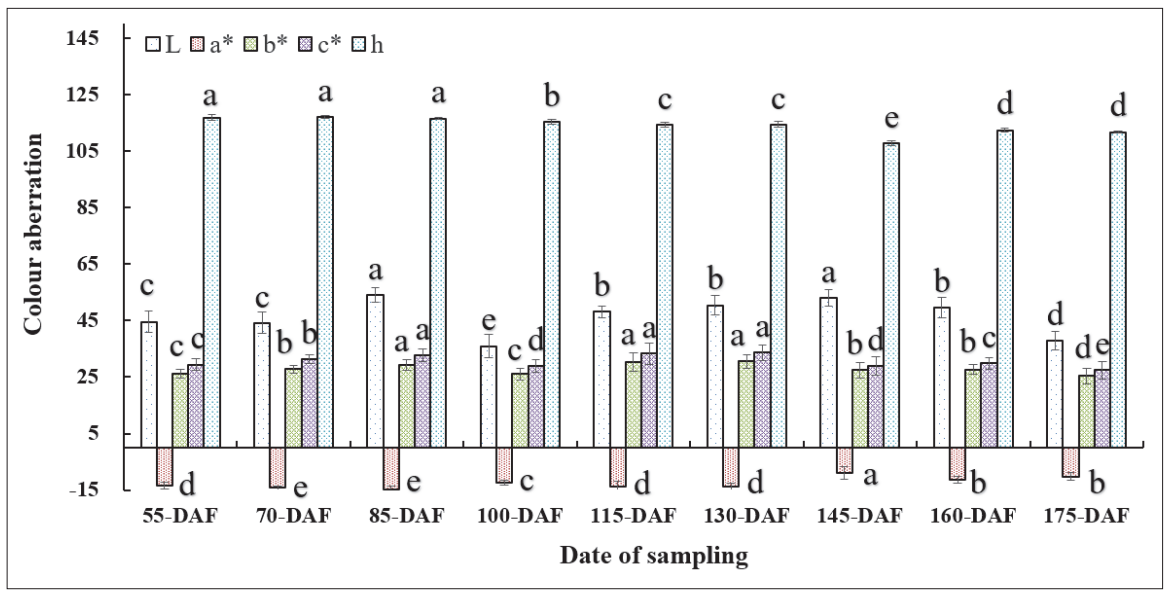

FIGURE 8. Changes of flesh color during fruits development.

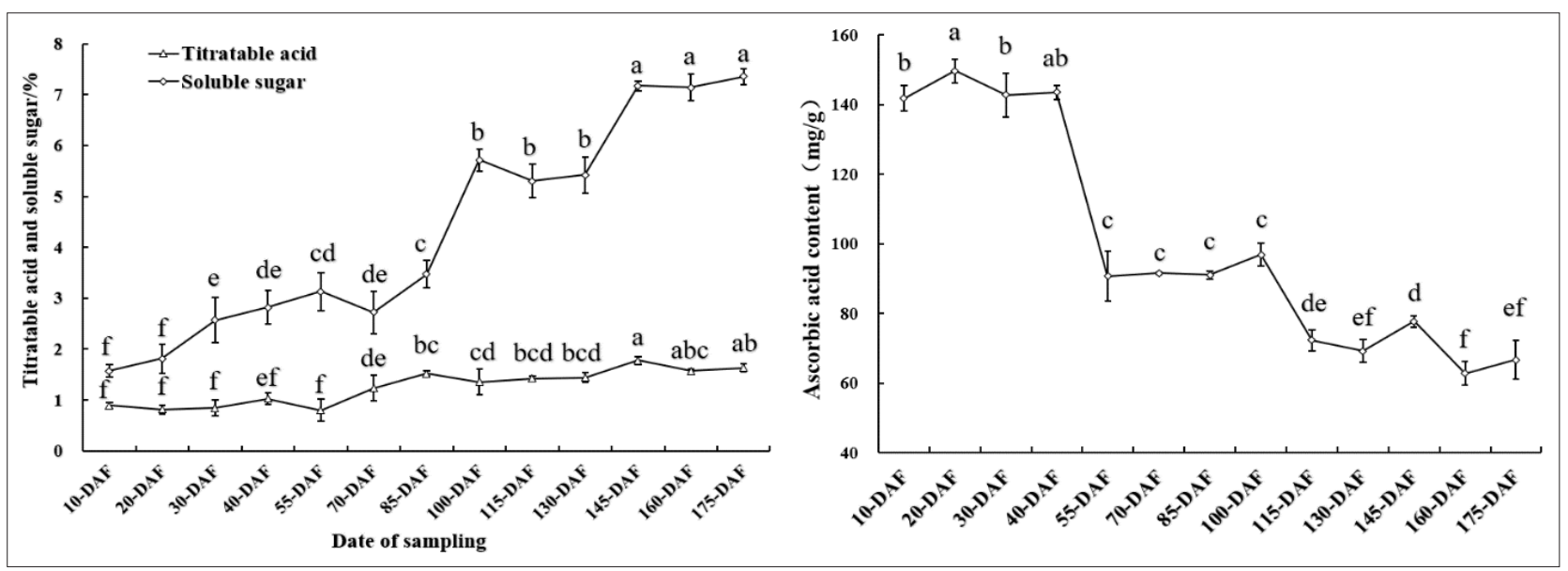

FIGURE 9. Changes of titratable acid, soluble sugar and ascorbic acid content of 'Fenghuang 1' during fruits development. 


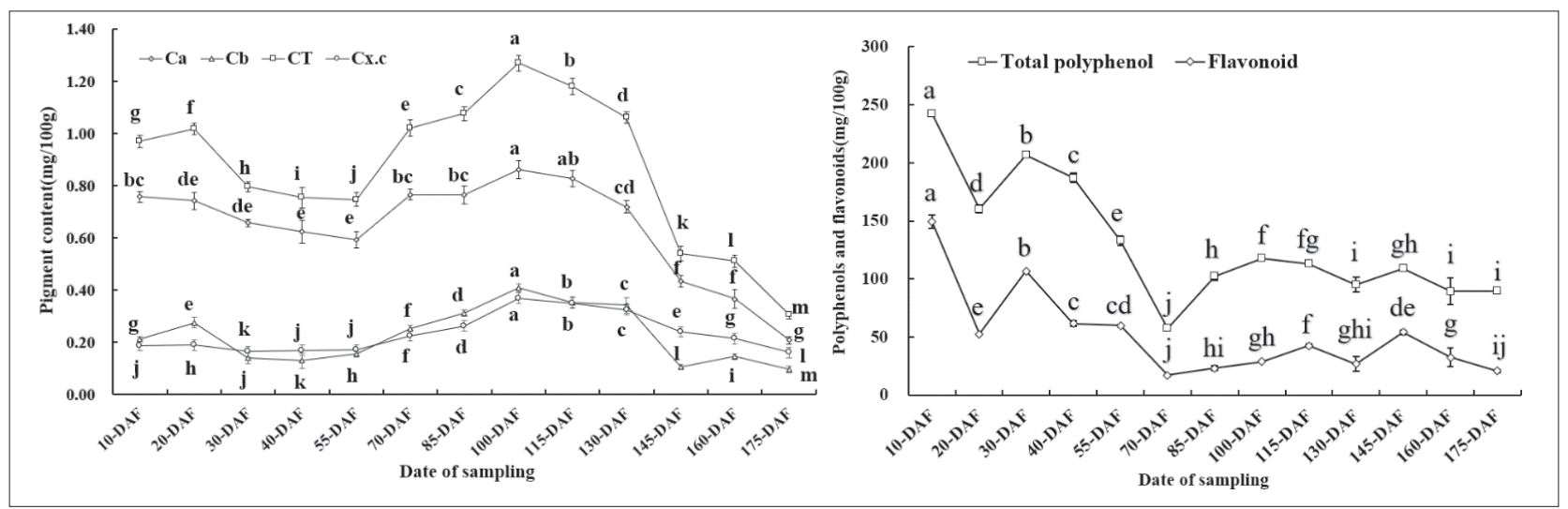

FIGURE 10. Changes of pigment, flavonoid and polyphenol of 'Fenghuang 1' during fruits development.
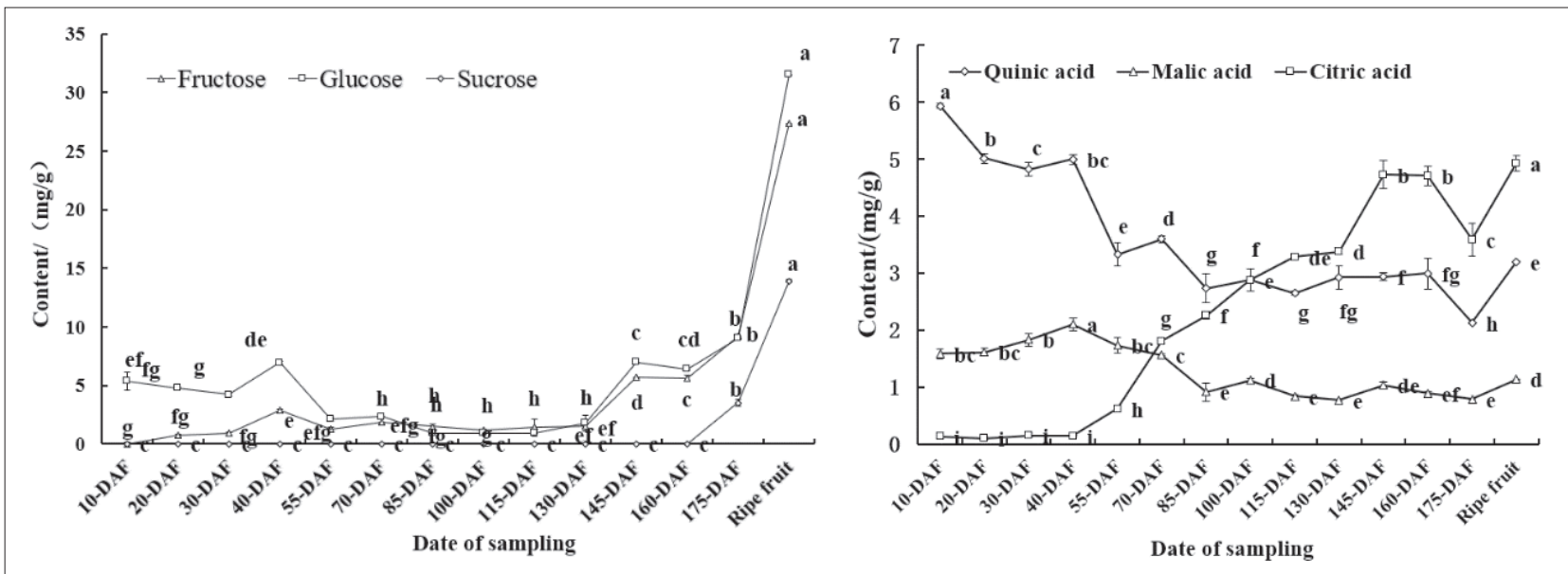

FIGURE 11. Changes of sugar and acid compositions of 'Fenghuang 1' during fruits development.

DAF $70 \mathrm{~d}-100 \mathrm{~d}$ and $130 \mathrm{~d}-175 \mathrm{~d}$, it eventually reached $8.776 \%$. The content of ascorbic acid was highest in young fruit and decreased throughout the development of the fruit (Figure 9).

6. Pigment, flavonoid and polyphenol. The content of each pigment of 'Fenghuang 1 ' showed a downward trend before DAF $55 \mathrm{~d}$, and then increased rapidly during DAF $50 \mathrm{~d}-100 \mathrm{~d}$. There was twice descent process after DAF $100 \mathrm{~d}$, the first was a rapid descent process during DAF $100 \mathrm{~d}-145 \mathrm{~d}$, the other was a slow descent process during DAF 145 d-175 d (Figure 10). During fruit development, the content of flavonoid and total polyphenols showed the same change trend, both of which showed a decreasing trend. In the first period of fruit growth, the content of flavonoid and total polyphenol showed a decreasing trend, while in the second period of fruit growth, the content fluctuated up and down (Figure 10). The content of total polyphenols was always higher than flavonoid.

7. Correlation analysis of various indexes. The correlation analysis of various indexes showed that weight was positively correlated with soluble sugar and titratable acid content, and weight was negatively correlated with flavonoids, total polyphenols and ascorbic acid. There was a significant positive correlation between flavone content, total polyphenol content and ascorbic acid content, while there was significant negative correlation between soluble sugar content and titratable acid content. There was a significant positive correlation between total polyphenol content and ascorbic acid content, and a significant negative correlation between total polyphenol content and soluble sugar, titratable acid content. There also was a significant positive correlation between soluble sugar content and titratable acid content (Table 3).

TABLE 3. Correlation analysis of various indexes of 'Fenghuang 1' during fruit growth and development. ** Highly significant difference $>0.01$.

\begin{tabular}{|c|c|c|c|c|c|c|}
\hline & Weight & Flavonoid & Total polyphenol & Ascorbic acid & Soluble sugar & Titratable acid \\
\hline Weight & 1 & & & & & \\
\hline Flavonoid & $-0.714^{* *}$ & 1 & & & & \\
\hline Total polyphenol & $-0.800^{* *}$ & $0.922^{* *}$ & 1 & & & \\
\hline Ascorbic acid & $-0.930^{* *}$ & $0.673^{* *}$ & $0.838^{* *}$ & 1 & & \\
\hline Soluble sugar & $0.897^{\star \star}$ & $-0.540^{\star *}$ & $-0.618^{* *}$ & $-0.810^{* *}$ & 1 & \\
\hline Titratable acid & $0.808^{* *}$ & $-0.579^{* *}$ & $-0.667^{* *}$ & $-0.755^{\star \star}$ & $0.806^{* *}$ & 1 \\
\hline
\end{tabular}




\section{Sugar and acid compositions date}

Change of sugar and acid compositions showed glucose and fructose fluctuate less before DAF $130 \mathrm{~d}$, then glucose and fructose gradually increased, while sucrose only began to appear at DAF $160 \mathrm{~d}$. The content of malic acid and quinic acid gradually decreased and citric acid increased during the whole growth period (Figure 11).

\section{Discussion}

Novel kiwifruit cultivar 'Fenghuang 1' displayed improved fruit quality compared to parent fruit ('Jinfeng') based on fruit dry matter and soluble solids content. Actually, there was significant difference between 'Fenghuang 1 ' and 'Hort16A' on soluble solids content and dry matter. This content of 'Fenghuang 1 ' was higher than that of parent (Jinfeng) and 'Hort16A' after room temperature storage, not only in this experiment, but also in previous studies (Burdon et al., 2014). According to the literature, ripe kiwifruit fruit soluble solids content of $14.00 \%$ or higher is necessary for obtaining satisfactory consumer acceptance (Jaeger et al., 2011). All fruits of 'Fenghuang 1' attained this level, whether ripe or after room temperature storage; none of the fruits of 'Jinfeng' reached this level. In addition, the dry matter of 'Fenghuang 1 ' fruits is above $18.00 \%$, it has an important effect on the flavor quality (Nardozza et al., 2011). Combining the results of the economic characters, molecular and ploidy level, 'Fenghuang 1 ' could be identified as a novel kiwifruit cultivar.

The economic characteristics of fruit were evaluated systematically; we found that the growth period of fruit was $175 \mathrm{~d}$ and was formal "double S" curve, this was similar to 'Jintao' and 'Jinfeng' (Zhong et al., 2015; Gao and Tao, 1994). The soluble solids content and dry matter content increased rapidly after the color of the seed turned brown; moreover, the fruit reached the requirement of soluble solids for harvesting at DAF $145 \mathrm{~d}$. The development of seed can promote the development of fruit, it is consistent with previous research results (Lai et al., 1989). The content of soluble solid and dry matter all decreased at DAF $115 \mathrm{~d}$, it may be the result of the ratio of the soluble sugar content to titratable acid going down. After that, the rate of growth of soluble sugar content is much higher than the rate of growth of titratable acid, which increases the ratio. And that's why the content of soluble solid and dry matter keeps going up after DAF $115 \mathrm{~d}$. In addition, the sugar-acid ratio is one of the important indexes affecting fruit quality (Macrae et al., 2010). During the whole growth period, the contents of flavonoids, total polyphenols and ascorbic acid content were all in a downward trend, which may be related to the activity of enzymes during the growth period; it requires further research by combining biochemical and molecular biological means (Yuan et al., 2016; Hou et al., 2009). The correlation analysis of each index is basically consistent with previous research (Wang et al., 2017; Jin et al., 2016). It is worth mentioning that we find that flavonoids, total polyphenols and ascorbic acid content show very significant positive correlation with each other.

Different genotype kiwifruit have different accumulation mechanisms of sugar and acid compositions, and the main types of sugar and acid are also different (Marsh et al., 2009; Nishiyama et al., 2008). Compared with previous studies, we found that the content of organic acid was lower than other yellow flesh kiwifruit, such as the content of malic acid and quinine was lower than previous studies (Wang et al., 2017). The 'Fenghuang 1' maybe the higher-sugar and lower-acid type of kiwifruit. The main types of sugar were fructose and glucose, the main types of acid were citric acid and quinine acid.

\section{Conclusions}

'Fenghuang 1' kiwifruit is a novel mid-maturing cultivar with high dry matter content from seedlings of $A$. chinensis 'Jinfeng', differentiates itself due to its morphological characteristics and DNA profile as determined by SSR molecular analysis. The novel cultivar is described by specific characteristics such as high fruit dry matter content. The ploidy level is tetraploid, and more resistant to canker than parent and 'Hort16A'. The novel kiwifruit cultivar is a kind of higher-sugar and lower-acid kiwifruit, the main types of sugar were fructose and glucose, the main types of acid were citric acid and quinine acid. The discovery and analysis of mid-maturing cultivar with high dry matter content would extend fruit supply period of $A$. chinensis, and contribute to our understanding of the regulatory mechanism of dry matter in A. chinensis and more generally in kiwifruit.

\section{Acknowledgments}

This work was supported by the National Natural Science Foundation of China (Grant No. 31760559, 31760567), the Jiangxi Provincial Department of Science \& Technology (Grant No. 20161ACF60007) and the Jiangxi Provincial Construction of Kiwifruit Industry Technology System (Grant No. JXARS-05).

\section{Competing interests}

The authors declare that they have no competing interests.

\section{Authors' contributions}

Xiaobiao Xu, Shanggao Zhao and Jianhua Leng conceived the study, Guiqing Tu and Bangming Li collected seeds and sown. Junjie Tao and Xueyan Qu recorded the phenological period. Chunhui Huang and Min Zhong collected materials and assisted in SSR analysis and ploidy. Guanglian Liao performed fruit quality analysis and wrote the manuscript. All authors have read and approved the final text.

\section{References}

Atkinson, R.G., and Macrae, E.A. (2007). Kiwifruit. Biotechnol. in Agric. \& Forestry 60, 329-346. https://doi.org/10.1007/978-3-54049161-3_13.

Burdon, J., Pidakala, P., Martin, P., McAtee, P.A., Boldingh, H.L., Hall, A., and Schaffer, R.J. (2014). Postharvest performance of the yellow-fleshed 'Hort16A' kiwifruit in relation to fruit maturation. Postharv. Biol. \& Technol. 92, 98-106. https://doi.org/10.1016/j. postharvbio.2014.01.004.

Gao, J.F. (2006). Experimental Instruction in Plant Physiology (Higher Education Press, in press).

Gao, L.P., and Tao, H.Z. (1994). Growth and development of kiwifruit. Acta Hortic. Sinica 4, 334-338.

Hou, C.M., Li, M.J., Ma, F., Liang, D., and Du, G.R. (2009). Changes of product accumulation and related enzyme activities in AsA metabolism during kiwifruit growth and development. Acta Hortic. Sinica 36(9), 1269-1276.

Huang, W.G., Cipriani, G., Morgante, M., and Testolin, R. (1998). Microsatellite DNA in Actinidia chinensis: isolation, characterisation, and homology in related species. Theor. Appl. Gen. 97(8), 12691278. https://doi.org/10.1007/s001220051019.

Jaeger, S.R., Harker, R., Triggs, C.M., Gunson, A., Campbell, R.L., Jackman, R., and Requejo-Jackman, C. (2011). Determining consumer purchase intentions: the importance of dry matter, size, and price of kiwifruit. J. Food Sci. 76(3), S177-S184. https://doi.org/10.1111/ j.1750-3841.2011.02084.x. 
Jin, F.L., et al. (2016). The correlation of different weight of kiwi on quality change in maturation period. Acta Agric. Boreali-Sinica 31(s1), 219-228.

Koh, Y.J., Kim, G.H., Jung, J.S., Lee, Y.S., and Hur, J.S. (2010). Outbreak of bacterial canker on Hort16A (Actinidia chinensis Planchon) caused by Pseudomonas syringae pv. actinidiae in Korea. N. Z. J. Crop \& Hortic. Sci. 38(4), 275-282. https://doi.org/10.1080/01140671.20 10.512624 .

Lai, R., Woolley, D.J., and Lawes, G.S. (1989). Retardation of fruit growth of kiwifruit (Actinidia deliciosa) by leaves: Interactions with vine performance and seed number. Sci. Hortic. 39(4), 319-329. https://doi.org/10.1016/0304-4238(89)90125-8.

Macrae, E.A., Bowen, J.H., and Stec, M.G.H. (2010). Maturation of kiwifruit (Actinidia deliciosa cv. Hayward) from two orchards: Differences in composition of the tissue zones. J. Sci. Food \& Agric. 47(4), 401-416. https://doi.org/10.1002/jsfa.2740470403.

Marsh, K.B., Boldingh, H.L., Shilton, R.S., and Laing, W.A. (2009). Changes in quinic acid metabolism during fruit development in three kiwifruit species. Funct. Plant Biol. 36, 463-470. https://doi. org/10.1071/FP08240

Mavromatis, A.G., Arvanitoyannis, I., Nanos, G., Sakellariou, M., Ilanidis, C., and Korkovelos, A. (2010). Molecular fingerprinting of a new Kiwifruit cultivar (cv. Tsehelidis) and comparative analysis with cv. Hayward according to physicochemical properties. Sci. Hortic. 125(3), 277-282. https://doi.org/10.1016/j.scienta.2010.03.010.

Nardozza, S., Gamble, J., Axten, L.G., Wohlers, M.W., Clearwater, M.J., Feng, J., and Harker, F.R. (2011). Dry matter content and fruit size affect flavour and texture of novel Actinidia deliciosa genotypes. J. Sci. Food \& Agric. 91(4), 742-748. https://doi.org/10.1002/jsfa.4245.

Nishiyama, I., Fukuda, T., Shimohashi, A., and Oota, T. (2008). Sugar and organic acid composition in the fruit juice of different Actinidia varieties. Food Sci. \& Technol. Int. Tokyo 14(1), 67-73. https://doi. org/10.3136/fstr.14.67.

Niu, J., Zhao, J., Wu, B., Li, S., Liu, G., and Jiang, Q. (2006). Sugar and acid contents in peach and nectarine derived from different countries and species. Acta Hortic. Sinica 33(1), 9-14.

Seal, A.G. (2003). Plant breeding challenges to making kiwifruit a worldwide mainstream fresh fruit. Acta Hortic. 610, 75-80. https:// doi.org/10.17660/ActaHortic.2003.610.7.

Wang, G., et al. (2017). The components and contents of organic acid in kiwifruit fruits from different cultivars. J. Agric. 7(12), 81-84.

Wang, M.S., et al. (2017). Differences of fruit quality among varieties of raspberry crop in saline-alkali soils. J. Tianjin Agric. College 24(2), 14-18.

Wu, J.H., Ferguson, A.R., Murray, B.G., Jia, Y., Datson, P.M., and Zhang, J. (2012). Induced polyploidy dramatically increases the size and alters the shape of fruit in Actinidia chinensis. Annals of Botany 109(1), 169-179. https://doi.org/10.1093/aob/mcr256.

Yuan, Y.L., et al. (2016). Difference of ascorbic acid synthesis and metabolism in different genotypes of kiwifruit. Plant Physiol. J. 12, 1877-1883.

Zhong, C.H., et al. (2015). Studies on characterization of fruit development of interspecific hybrid cultivar - 'Jinyan'. J. Fruit Sci. 32(6), 1152-1160.

Received: May 9, 2019

Accepted: Jul. 22, 2019
Addresses of authors:

Guanglian Liao ${ }^{1}$, Xiaobiao $\mathrm{Xu}^{1, *}$, Min Zhong ${ }^{1}$,

Chunhui Huang ${ }^{1}$, Guiqing $\mathrm{Tu}^{2}$, Bangming $\mathrm{Li}^{2}$, Junjie $\mathrm{Tao}^{1}$,

Xueyan $\mathrm{Qu}^{1}$, Shanggao $\mathrm{Zhao}^{2}$ and Jianhua Leng ${ }^{2}$

${ }^{1}$ Kiwifruit Institute of Jiangxi Agricultural University, Nanchang Jiangxi, 330045, P.R. China

${ }^{2}$ Kiwifruit Institute of Fengxin County in Jiangxi Province, Yichun Jiangxi, 330700, P.R. China

* Corresponding author; E-mail: xbxu@jxau.edu.cn Tel.: +8613767008891 